\title{
BMJ Open SurgiCal Obesity Treatment Study (SCOTS): protocol for a national prospective cohort study of patients undergoing bariatric surgery in Scotland
}

Jennifer Logue, ${ }^{1}$ Sally Stewart, ${ }^{2}$ Jane Munro, ${ }^{1}$ Julie Bruce, ${ }^{3}$ Eleanor Grieve, ${ }^{2}$ Mike Lean, ${ }^{4}$ Robert S Lindsay, ${ }^{1}$ Duff Bruce, ${ }^{5}$ Abdulmajid Ali, ${ }^{6}$ Andrew Briggs, ${ }^{2}$ Naveed Sattar, ${ }^{1}$ lan Ford, ${ }^{7}$ on behalf of the SCOTS Investigators

To cite: Logue J, Stewart S, Munro J, et al. SurgiCal Obesity Treatment Study (SCOTS): protocol for a national prospective cohort study of patients undergoing bariatric surgery in Scotland. BMJ Open 2015;5:e008106. doi:10.1136/bmjopen-2015008106

- Prepublication history for this paper is available online. To view these files please visit the journal online (http://dx.doi.org/10.1136/ bmjopen-2015-008106)

Received 4 March 2015 Accepted 14 April 2015

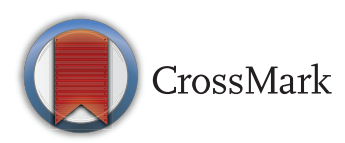

For numbered affiliations see end of article.

Correspondence to Dr Jennifer Logue; Jennifer. Logue@Glasgow.ac.uk

\section{ABSTRACT}

Introduction: The efficacy of bariatric surgery for large-scale, long-term weight loss is well established. However, many questions remain over the continual benefits and cost-effectiveness of that weight loss for overall health, particularly when accounting for potential complications and adverse events of surgery. Health research institutes in the UK and the USA have called for high-quality longitudinal cohort studies of patients undergoing bariatric surgery, assessing outcomes such as surgical complications, mortality, diabetes remission, microvascular complications, cardiovascular events, mental health, cost and healthcare use.

Methods and analysis: SurgiCal Obesity Treatment Study (SCOTS) is a national, prospective, observational, cohort study of patients undergoing primary bariatric surgical procedures in Scotland. This study aims to recruit 2000 patients and conduct a follow-up for 10 years postbariatric surgery using multiple data collection methods: surgeon-recorded data, electronic health record linkage, and patient-reported outcome measures. Outcomes measured will include: mortality, weight change, diabetes, surgical, cardiovascular, cancer, behavioural, reproductive/urological and nutritional variables. Healthcare utilisation and economic productivity will be collected to inform costeffectiveness analysis.

Ethics and dissemination: The study has received a favourable ethical opinion from the West of Scotland Research Ethics committee. All publications arising from this cohort study will be published in open-access peer-reviewed journals. All SCOTS investigators (all members of the research team at every recruiting site) will have the ability to propose research suggestions and potential publications using SCOTS data; a publications committee will approve all requests for use of SCOTS data and propose writing committees and timelines. Lay-person summaries of all research findings will be published simultaneously on the SCOTS website (http://www. scotsurgeystudy.org.uk).
Strengths and limitations of this study

- The major advantage of SurgiCal Obesity Treatment Study (SCOTS) is the availability of high-quality health record data recorded in a standardised manner across Scotland.

- Inclusion of all state-funded and private hospitals undertaking bariatric surgery ensures geographical representation.

- SCOTS will allow detailed follow-up of clinical, surgical outcomes and changes in health status after bariatric surgery, underpinned with a detailed cost-effectiveness analysis based on reallife care and treatment practices.

- The design of the study means that participants will be able to be followed through record-linkage for many decades, until death, at minimal additional cost. All patients can be followed, therefore removing any potential for bias from/to participant drop-out due to poor surgical outcome or ill health.

- The major limitation of SCOTS is the relatively low numbers of bariatric surgery procedures performed in Scotland per head of population, and the challenge of low volume centres compared with international standards. However, the data collection has been designed with this in mind to ensure that surgeon and centre experience is captured, and this variety will allow surgical experience to be explored as an explanatory variable.

\section{INTRODUCTION}

The efficacy of bariatric surgery for large-scale, long-term weight loss is well established. ${ }^{1}$ However, many questions remain over the continual benefits of that weight loss for overall health, particularly when accounting for potential complications of surgery. In 2009, Picot $e t a l^{2}$ published a systematic review and economic evaluation of the clinical and cost-effectiveness of bariatric surgery for obesity, funded by the UK's 
National Institute for Health Research (NIHR) Health Technology Assessment Programme (HTA). The conclusion was that high-quality data were lacking and further research was required, particularly large-scale cohort studies to ascertain patient quality of life, late complications requiring reoperation, the effect of surgeon experience, duration of comorbidity remission and the full use of health and care resources to allow comprehensive cost-effectiveness analysis.

In May 2013, the USA National Institute for Diabetes and Digestive and Kidney Diseases and the National Heart, Lung and Blood Institute held a multidisciplinary workshop on the long-term outcomes of bariatric surgery, and reached similar conclusions regarding research priorities to that of Picot et al. ${ }^{3}$ The main areas highlighted as having significant knowledge gaps included the incidence of surgical complications, overall mortality, duration of type 2 diabetes remission and microvascular complications, cardiovascular events, mental health outcomes, malignancies, fertility and fetal outcomes, and overall cost and healthcare use. This workshop agreed that there should be investment in high-quality, observational studies, potentially utilising electronic health records where available.

In response to the Picot systematic review, in 2010, UK NIHR HTA issued an open call for research proposals for a long-term longitudinal cohort study of patients undergoing bariatric surgery in the UK, with a minimum of 10 years follow-up. The outcomes were to include quality of life, survival, weight, BMI, diabetes and surgical complications. The SurgiCal Obesity Treatment Study (SCOTS) was funded in 2011. This paper describes the study design and outcomes.

\section{OBJECTIVES}

The overall objective of SCOTS is to establish the clinical outcomes and adverse events of different bariatric surgical procedures, their impact on quality of life and nutritional status, and the effect on comorbidities in the short and long term in a cohort of over 2000 patients.

The specific objectives are to establish in a cohort of obese patients who are undergoing bariatric surgery

- All-cause and cause-specific mortality over a mean of 10 years since bariatric surgery.

- Incidence of cardiovascular disease, cancer and diagnosis of diabetes over a mean of 10 years since bariatric surgery.

- Incidence of acute and chronic postoperative complications. Acute complications, defined as up to 3 months postsurgery, will include surgical site infection; chronic complications will include revisional surgery, plastic surgery and chronic pain.

- Change in health-related quality of life, anxiety and depression over time, preoperatively and postoperatively, for a mean of 10 years from the date of bariatric surgery.
- The micronutrient and weight status preoperatively and postoperatively for a mean of 10 years since bariatric surgery.

- The glycaemic control, lipids, blood pressure, medication prescription and rate of diabetes complications (microalbuminuria and renal disease, retinopathy and foot ulceration) in those that have pre-existing diabetes or develop diabetes during a mean of 10 years of follow-up since bariatric surgery.

- Explanatory variables will be explored for each outcome.

\section{METHODS}

Study design

SCOTS is a national, prospective, observational cohort study of patients undergoing primary bariatric surgical procedures in Scotland. A total of 2000 patients will be recruited over a 5-year period from 2014 to 2019, and will be followed for a mean follow-up period of 10 years postsurgery.

\section{Participant and centre eligibility}

Patients scheduled to undergo bariatric surgery at any state-funded or private hospital in Scotland will be eligible for entry into the cohort. Currently, 10 statefunded and 4 private hospitals across Scotland provide bariatric surgery, and are thus eligible to participate.

For the purposes of SCOTS, a bariatric surgical procedure is defined as 'any surgical intervention which has the primary purpose of large-scale weight loss in a patient who is obese'. Eligible procedures include Roux-en-Y gastric bypass (RYGB), sleeve gastrectomy (SG) and laparoscopic adjustable gastric banding (LAGB). The inclusion of emerging techniques in this definition will be considered by the Steering Committee throughout the duration of the cohort. At current rates for Scotland, it is anticipated that this may result in $60 \%$ of participants having SG, 30\% LAGB, and 20\% RYGB, but changing trends in the type of surgery offered may alter these predicted patterns.

SCOTS has received a favourable ethical opinion from the West of Scotland Research Ethics Committee, and full management approval at each hospital site.

\section{Outcomes}

The outcomes to be measured include mortality, weight, diabetes, surgical complications, cardiovascular, cancer, behavioural, reproductive/urological and nutritional outcomes. Other healthcare resource use of variables will be collected to inform a cost-effectiveness analysis. Outcomes are summarised in table 1.

\section{Data collection}

Data will be obtained from three main sources: the surgical team, patients, and from electronic health records (table 2). For some outcomes, multiple sources will be used to collect similar data to ensure overall 
Table 1 Surgical obesity treatment study outcomes

\begin{tabular}{|c|c|}
\hline Outcome category & Specific outcomes \\
\hline Mortality & 30 day, 1 year and 10 year mortality; all-cause and cause-specific \\
\hline Weight & Change in weight/body mass index \\
\hline \multirow[t]{9}{*}{ Surgical } & Incidence of surgical complications 1 year and 10 years postoperatively \\
\hline & Hospital length of stay/rate of readmission \\
\hline & Incidence of surgical site infection \\
\hline & Postoperative pain \\
\hline & Rate of reoperation \\
\hline & Rate of revisional procedures \\
\hline & Rate of body-contouring surgery \\
\hline & Incidence of excess skin affecting daily life \\
\hline & Change in gastrointestinal symptoms \\
\hline \multirow[t]{3}{*}{ Cardiovascular } & Incidence of fatal and non-fatal cardiovascular events \\
\hline & Incidence of fatal and non-fatal coronary heart disease \\
\hline & Cardiovascular medication prescription \\
\hline \multirow[t]{9}{*}{ Diabetes } & Incidence of type 2 diabetes \\
\hline & Change in glycaemic control \\
\hline & Change in blood lipid concentration \\
\hline & Change in blood pressure \\
\hline & Change in estimated glomerular filtration rate and microalbuminuria \\
\hline & Incidence of retinopathy \\
\hline & Incidence of amputation \\
\hline & Incidence of chronic kidney disease \\
\hline & Diabetes medication prescription \\
\hline Cancer & Incidence of all and specific cancers \\
\hline \multirow[t]{7}{*}{ Behavioural } & Change in prevalence/incidence of depression and anxiety \\
\hline & Change in health-related quality of life \\
\hline & Change in rate of alcohol use disorder \\
\hline & Change in smoking status \\
\hline & Change in physical activity levels \\
\hline & Change in fat, fibre and calcium intake \\
\hline & Life orientation (optimism) \\
\hline \multirow[t]{5}{*}{ Reproductive/urological } & Change in menstrual cycle/abnormalities \\
\hline & Incidence of pregnancy and fetal outcome \\
\hline & Change in prevalence of incontinence \\
\hline & Change in prevalence of prostatic symptoms \\
\hline & Change in rate of erectile dysfunction \\
\hline \multirow[t]{2}{*}{ Nutritional } & Incidence of micronutrient deficiency \\
\hline & Prescription of multivitamins and nutritional supplements \\
\hline \multirow[t]{8}{*}{ Economic } & Cost of presurgical and postsurgical care pathways \\
\hline & Healthcare utilisation \\
\hline & Change in receipt of Social Security benefits \\
\hline & Change in employment status \\
\hline & Change in education \\
\hline & Sickness absence from work/study \\
\hline & Change of use of aids and specialist equipment (eg, walking frame or stair lift) \\
\hline & Social care requirements \\
\hline Fractures & Incidence of fractures by site \\
\hline
\end{tabular}

completeness of the data set (eg, revisional surgery will be collated from clinical teams, self-reported by patients and from electronic health records; fractures will be reported through either hospital and emergency department admissions, or through radiology reports).

Where possible, definitions and data sources have been kept consistent with the UK National Bariatric Surgery Register ${ }^{4}$ and the NIH-funded Longitudinal Assessment of Bariatric Surgery ${ }^{5}$ to allow comparison and meta-analysis of outcomes.

\section{Recruitment procedures and consent}

Patients are approached about the study at least 4 weeks prior to their primary bariatric surgical procedure. This is undertaken by either the clinical bariatric surgery team or by a research nurse in preoperative assessment clinics. Patients are shown an $8 \mathrm{~min}$ information film and provided with an information booklet explaining the SCOTS. Individual participant consent is obtained on a subsequent clinical visit. Informed consent is sought at three levels, for (1) access to medical records 
and linkage of their electronic health records, (2) postal questionnaires preoperatively and postoperatively and (3) contact for future studies. Each level of consent is dependent on the previous level, for example, postal (or electronic) questionnaires are not sent without consent to link electronic health records.

Table 2 Surgical obesity treatment study data collection

\begin{tabular}{|c|c|c|}
\hline Data collection & Type & Questionnaire \\
\hline \multicolumn{3}{|l|}{ Surgical data } \\
\hline & \multirow[t]{8}{*}{ Primary procedure } & Surgeon \\
\hline & & Procedure \\
\hline & & $\begin{array}{l}\text { Operative details (including band type, staple type, approach, dissection, } \\
\text { biliopancreatic limb length) }\end{array}$ \\
\hline & & Presurgical intervention (including balloon or endobarrier) \\
\hline & & Patient weight day of operation \\
\hline & & American Society of Anesthesiologists (ASA) grade \\
\hline & & Operation performed by surgeon in training \\
\hline & & Perioperative blood transfusion \\
\hline & \multirow{2}{*}{ Reoperations } & Reoperation (detail as per primary procedure) \\
\hline & & Reason for reoperation \\
\hline & Surgical experience & Surgeon experience (baseline, updated annually) \\
\hline \multicolumn{3}{|c|}{ Patient-reported outcome measures } \\
\hline & \multirow[t]{5}{*}{ Demographics } & ID, sex, age \\
\hline & & Marital status \\
\hline & & Ethnicity \\
\hline & & Education \\
\hline & & Occupation \\
\hline & \multirow[t]{4}{*}{ Medical history } & Medical history $^{8}$ \\
\hline & & Weight history ${ }^{9}$ \\
\hline & & Family history (diabetes, coronary heart disease (CHD)) \\
\hline & & Family history (weight) \\
\hline & \multirow{4}{*}{$\begin{array}{l}\text { Quality of life-Health } \\
\text { related }\end{array}$} & $\mathrm{SF} 12^{10}$ \\
\hline & & EQ5D $5 L^{11}$ \\
\hline & & Patient Health Questionnaire (PHQ9) ${ }^{12}$ \\
\hline & & Generalised Anxiety Disorder (GAD7) $^{13}$ \\
\hline & \multirow[t]{6}{*}{ Impact of weight } & Impact of Weight on Quality of Life (IWQOL)-Lite ${ }^{14}$ \\
\hline & & Reproductive health (female) ${ }^{5}$ \\
\hline & & $\begin{array}{l}\text { Erectile dysfunction-Massachusetts Male Aging Study: Single question } \\
\text { assessment (male) }{ }^{15}\end{array}$ \\
\hline & & International Prostate Symptom Score ${ }^{16}$ \\
\hline & & $\begin{array}{l}\text { International Consultation on Incontinence Questionnaire Urinary Incontinence } \\
\text { Short Form }{ }^{17}\end{array}$ \\
\hline & & Modified Reflux questionnaire ${ }^{18}$ \\
\hline & \multirow[t]{5}{*}{ Lifestyle } & Smoking \\
\hline & & Alcohol Use Disorders Identification Test: Self-Report Version ${ }^{19}$ \\
\hline & & $\begin{array}{l}\text { International Physical Activity Questionnaire short last } 7 \text { days self-administered } \\
\text { format }^{20}\end{array}$ \\
\hline & & Dietary Fat and Fibre intake \\
\hline & & Dietary Intervention in Primary Care (DINE) ${ }^{21}$ \\
\hline & \multirow[t]{2}{*}{ Personal outlook } & Life orientation test (revised) ${ }^{22}$ \\
\hline & & Expectations of surgery-Goals and Relative Weights Questionnaire ${ }^{23}$ \\
\hline & \multirow[t]{4}{*}{ Surgical recovery } & Postoperative pain \\
\hline & & Surgical site infection ${ }^{24}$ \\
\hline & & Skin excess \\
\hline & & Body contouring surgery ${ }^{25}$ \\
\hline & \multirow[t]{5}{*}{ Healthcare usage } & Outpatient care ${ }^{26}$ \\
\hline & & Contacts with other health and social care professionals \\
\hline & & Devices and specialist equipment ${ }^{27}$ \\
\hline & & Benefits \\
\hline & & Multivitamins and supplements use \\
\hline
\end{tabular}




\begin{tabular}{|c|c|c|}
\hline Data collection & Type & Questionnaire \\
\hline \multirow[t]{9}{*}{$\begin{array}{l}\text { Electronic health } \\
\text { records }\end{array}$} & Hospital admissions & $\begin{array}{l}\text { Diagnosis, length of stay, intensive/high dependency, in-hospital mortality } \\
\text { Scottish Morbidity Record } 01\end{array}$ \\
\hline & Maternity & $\begin{array}{l}\text { Inpatient and day case including birth weight, gestational age, mode of delivery, } \\
\text { induction, pregnancy outcome and neonatal care }\end{array}$ \\
\hline & & Scottish Morbidity Record 02/Scottish Birth Record \\
\hline & Psychiatric & $\begin{array}{l}\text { Inpatient care records-including diagnosis, treatment, length of stay } \\
\text { Scottish Morbidity Record } 04\end{array}$ \\
\hline & Malignancy & $\begin{array}{l}\text { Site, histology, stage, grade, treatment } \\
\text { Scottish Cancer Registry }\end{array}$ \\
\hline & Mortality & National Records of Scotland Death Records \\
\hline & Diagnostic tests & $\begin{array}{l}\text { Biochemistry, haematology, radiology, pathology and microbiology results } \\
\text { Scottish Care Information-Store }\end{array}$ \\
\hline & Diabetes & $\begin{array}{l}\text { Including date of diagnosis, type, treatment, retinal and foot screening } \\
\text { Scottish Care Information-diabetes }\end{array}$ \\
\hline & Medications & $\begin{array}{l}\text { Type and adherence-encashed prescriptions } \\
\text { Prescribing Information System }\end{array}$ \\
\hline
\end{tabular}

\section{Surgical data}

In order to encourage site participation and maintain interest in the study, data from the surgical clinical team is collected using a specifically designed, webbased, clinical information system which has a number of features of use to the clinical teams. At first log-in, the system records basic surgical data, including surgeon experience, local operative procedures performed at that site including the standard approach for each procedure performed by that surgeon (eg, make of staple or bands used). Details of consented individuals are entered by the central study centre, and the details of each patient are visible to that local clinical team allowing them to enter further preoperative and surgical details. This is kept simple by allowing some modification to the standard surgical details stored. SCOTS data collection system allows weight and band adjustments at follow-up visits to be recorded, as well as details of, and indications for, revisional surgery. If revisional surgery is being performed at another site, the patient's record can be identified on the system. A number of templates exist for clinical letters, patient reports and clinical notes which populate with details from the system and output as a portable document format (pdf) file for entry into hospital letters and records.

\section{Patient-reported outcome measures}

Participants are asked to complete a suite of questionnaires at a number of time points throughout the study: preoperatively and 1 month, 6 months, 1, 2, 3, 5, 7 and 10 years postoperatively. Questionnaires were developed in conjunction with patient involvement and were piloted (described below). Final questionnaires consist of validated scales covering a wide range of outcome measures. Participants have the option of completing these via a web-based system or on paper by post. They receive up to two reminders for each questionnaire. The full questionnaire takes around $1 \mathrm{~h}$ to complete, and start and finish dates are recorded to allow them to be completed over several days.

Questionnaires at all time points include weight, marital status, education, employment, general and obesity-specific quality of life, anxiety, depression, reproductive health, urological health, gastrointestinal symptoms, smoking, alcohol use, physical activity, diet, healthcare utilisation, social security and nutritional supplements. Baseline preoperative questionnaires include demographics, medical history, family history, weight history and expectations of surgery. Postoperative questionnaires include surgical recovery, pain intensity, surgical site infection, skin excess and plastic surgery.

\section{Electronic health records}

Scotland is fortunate to have excellent electronic health records. All patient healthcare interactions in Scotland are recorded by use of a single patient ID number. IT systems are common across all 14 health board areas, and a single government-funded department (Information Services Division) collates information for research purposes. This has allowed Scotland to become a leader in research using routine health records, and these systems are being utilised for follow-up in SCOTS.

Participants in SCOTS will be followed using multiple existing Scottish health record data sets: Scottish Morbidity Records-general acute/inpatient and day case (SMR 01), maternity inpatient and day case (SMR 02), and mental health inpatient and day case (SMR 04) data sets; National Records of Scotland Death Records; Scottish Cancer Registry; Scottish Care Information diabetes data set (SCI-Diabetes); Scottish Care Information Store (SCI-Store) diagnostic data set and the prescribing information system dataset.

Evidence of quality assurance for electronic health record data sets in Scotland is very high: an assessment 
of 2010/2011 data by comparison with local hospital case records showed an overall high degree of accuracy. For conditions of interest, this included accuracy of 97.3\% for ischaemic heart disease, $99.3 \%$ for fractures, 98.2\% for myocardial infarction and $98.4 \%$ for upper gastrointestinal endoscopy. ${ }^{6}$ Diabetes records are derived from all hospitals in Scotland and all of approximately 1000 primary care centres (except 5), which are downloaded to SCI-Diabetes on a daily basis. This dataset represents over $99 \%$ of people with diagnosed diabetes in Scotland.

\section{Treatment pathways}

Every site completes an annual survey to detail their current preoperative and postoperative care pathways. This includes how patients enter the surgical pathway (eg, direct or self-referral or via a non-surgical weight management programme), any presurgical requirements for weight loss and the standard numbers of appointments with dieticians, psychologists, surgeons and physicians that each patient receives. This information will allow outcomes from high-contact and low-contact presurgical and postsurgical care pathways to be compared.

\section{Health economics}

Health economist involvement in the design and development of the study has ensured that all relevant healthcare resource use data will be captured. Cost analysis will involve comparison of preoperative and postoperative care pathways at each site, and will include estimates of the costs of immediate ( 30 day) postoperative complications.

\section{Surgical learning curve}

The effect of the surgical learning curve on patient outcome is now well recognised. ${ }^{7}$ In order to account for this within analyses, surgeons will be asked to record date of completion of postgraduate surgical training and volume of bariatric surgery procedures performed (total, last 1 and 5 years). Total numbers of bariatric procedures (primary and revisional) performed by each individual surgeon in the previous year are also requested annually.

\section{Adverse outcome reporting}

Given the duration of this epidemiological study, there is a possibility that higher than expected adverse event rates may be detected, such as surgical site infection or 30-day surgical mortality. An adverse outcome plan has been agreed by the Steering Committee, and all participating surgeons have approved this plan. In preparation for each peer-reviewed publication, the study statisticians will generate reports to assess variability in outcomes between study sites, but these will be anonymised. No statistical analysis will be undertaken at individual surgeon level. Rates will be presented for all sites using funnel plots, with adjustment for case-mix, to identify sites that have outcome rates significantly above or below what would be expected given patient characteristics (which will be predefined in the statistical analysis plan). If a particular site is identified by the SCOTS team as having a potentially poorer outcome than expected, a letter will be sent from the Chief Investigator to the Principal Investigator and the Medical Director covering that site. An individual report will be prepared for that study site, summarising key data for that site, and including copies of the funnel plots from the main report, with the data point for that study site highlighted. Outcomes which will be considered eligible for assessment include rates of mortality, pulmonary thromboembolic disease, cardiovascular disease at 1 year, reoperations, surgical site infections, and nutritional deficiencies.

\section{Reference populations/comparator groups}

Collection of similar data from a non-surgical comparator group is not funded within the SCOTS, however, we have access to a number of possible reference populations. Electronic diabetes health record data sets allow an anonymous comparator group, matched for key demographics, to be created with prospective follow-up of diabetes care and complications compared to those with diabetes who had bariatric surgery. Also, several of the non-surgical weight management services within Scotland have detailed patient records again allowing the formation of anonymous comparator groups. Large cohorts, such as UK Biobank, also provide reference populations with prospective outcomes. In time, we hope to develop a similar cohort of patients undergoing lifestyle weight management with a comparable collection of baseline data, including psychological and mental health questionnaires, to help account for any biases within a self-selecting bariatric surgery population.

\section{Patient involvement}

We sought to form a patient public involvement (PPI) group to allow patient representation and input in the design of the study; public and patient involvement is encouraged by UK research councils, and mandatory for NIHR-funded research. A small focus group was formed comprising five patients who had previously undergone bariatric surgery in either the state-funded or private sector. The SCOTS PPI group has been consulted over a number of topics including: the information provided to patients about the study (patient information sheets, invitation letters and web site), content and design of electronic and paper patient-reported questionnaires, communication throughout the study, such as newsletters and reminder letters, and the need for incentives to take part and complete questionnaires. The focus group has met twice, each meeting lasting 3-4 h; participants have also been contacted by email periodically with additional questions.

\section{Development of patient questionnaires}

Piloting of patient questionnaires was undertaken during the development phase of the study. Some 
specific questions were considered by researchers to be of a sensitive nature (eg, sexual function, continence). However, these were not considered 'sensitive' by the SCOTS PPI focus group. Rather, items relating to diet, exercise and welfare benefits were considered to be particularly sensitive because they felt participants may feel 'judged'. To counter this, the PPI group requested the inclusion of a short explanatory sentence on why sections of questions were included. The group also identified important topics that were not included within the original question set, in particular, the inclusion of the need for dental work after bariatric surgery. Incentives were not felt to be necessary and were discounted in favour of a six monthly newsletter to include study progress, findings and words of continued thanks for participation.

\section{DISSEMINATION PLANS}

All publications arising from this cohort will be published in open-access peer-reviewed journals. All SCOTS investigators (all members of the research team at every recruiting site) will have the ability to propose research suggestions and potential publications using SCOTS data; a publications committee will approve all requests for use of SCOTS data and propose writing committees and timelines. Lay-person summaries of all research findings will be published simultaneously on the SCOTS website (http://www.scotsurgeystudy.org. uk). As is now recognised practice, we plan to make our data fully available for use in an anonymous form by other researchers at the end of the currently funded time period of this study (2027). Patient consent procedures cover the sharing of data for future research by external researchers. We will follow the NHS code of Practice on Protecting Patient Confidentiality issued by NHS Scotland. We will require a written request/proposal for data to be made to our publications committee who will need to be satisfied that all ethical approvals are in place and that funds are available to cover any costs of collating the necessary data. Regular reports of progress with the research will be required. The collaboration with the SCOTS study group should be acknowledged in the manuscript, normally by authorship stating the research workers 'on behalf of the SCOTS group'.

\section{ADVANTAGES AND LIMITATIONS}

The major advantage of SCOTS is the availability of high-quality health record data recorded in a standardised manner across Scotland. Inclusion of all statefunded and private hospitals undertaking bariatric surgery ensures geographical representation.

SCOTS will allow detailed follow-up of clinical, surgical outcomes and changes in health status after bariatric surgery, underpinned with a detailed cost-effectiveness analysis based on real-life care and treatment practices. The design of the study means that participants will be able to be followed through record-linkage for many decades, until death, at minimal additional cost. All patients can be followed, therefore removing any potential for bias from to participant dropout due to poor surgical outcome or ill health.

The major limitation of SCOTS is the relatively low numbers of bariatric surgery procedures performed in Scotland per head of population, and the challenge of low-volume centres compared with international standards. As a consequence, RYGB is the least commonly performed operation. These factors will be taken into account during analysis and compared with results from other studies. However, the data collection has been designed with this in mind to ensure that surgeon and centre experience is captured, and this variety will allow surgical experience to be explored as an explanatory variable.

\section{CONCLUSIONS}

SCOTS is the first national epidemiological study investigating long-term outcomes after bariatric surgery. This large data set will be one of the most detailed prospective cohort studies of bariatric surgery to date, made possible through linkage to high-quality, country-wide, electronic health records. SCOTS will assess important clinical, surgical and patient-reported outcomes over a 10-year period, including the incidence of, and risk factors for, surgical complications, overall mortality, diabetes remission, diabetes, microvascular complications, cardiovascular events, mental health, cancers, genitourinary and reproductive outcomes. This study will add substantially to the evidence base for the long-term effectiveness and cost-effectiveness of bariatric surgery outcomes.

\section{International implications}

No single study should be used to generate evidence for a commonly performed group of procedures that are the most effective treatment for a condition that effects $\sim 28 \%$ of people in the USA, and $\sim 22 \%$ of Europeans. We have developed a data set to allow important questions about bariatric surgery to be answered. However, bariatric surgery in Scotland is performed in low numbers compared with other countries; while we have excellent electronic data linkage, overall numbers of patients are low. Other sites may not have access to similar linked data, but do have higher numbers of patients. We would welcome the opportunity to collaborate with sites in other countries to compare patientreported data, and electronic health record data when available, to validate the generalisability of our detailed clinical outcome data for other populations.

\section{Author affiliations}

${ }^{1}$ Institute of Cardiovascular and Medical Sciences, University of Glasgow, Glasgow, UK

${ }^{2}$ Institute of Health and Wellbeing, University of Glasgow, Glasgow, UK

${ }^{3}$ Clinical Trials Unit, University of Warwick, Warwick, UK 
${ }^{4}$ School of Medicine, University of Glasgow, Glasgow, UK

${ }^{5} \mathrm{NHS}$ Grampian, Aberdeen, UK

${ }^{6}$ University Hospital Ayr, NHS Ayrshire and Arran, Ayr, UK

${ }^{7}$ Robertson Centre for Biostatistics, University of Glasgow, Glasgow, UK

Contributors JL conceived the study, and wrote the first draft of this paper. SS led the study design and setup. JM led the patient involvement. JB designed the patient-reported outcome measures. EG designed the data collection for economic analysis. ML and NS contributed to concept of the study and study design. RSL contributed to the concept of the study and study design, especially related to diabetes outcomes. DB contributed to the design of surgical data collection. $A A$ is the principal investigator at the recruiting site, contributed to the design of surgical data collection. $A B$ contributed to the study design, ensuring adequate data collected for economic evaluation. IF contributed to the concept of the study, study design and statistical analysis plan. All authors have reviewed and commented on this paper, approved the final version, and agree to be accountable for all aspects of the work in ensuring that questions related to the accuracy or integrity of any part of the work are appropriately investigated and resolved.

Funding This project is funded by the NIHR Health Technology Assessment Programme (project number 10/42/02) from January 2012 to July 2027.

Competing interests None declared.

Ethics approval West of Scotland Research Ethics Committee.

Provenance and peer review Not commissioned; peer reviewed for ethical and funding approval prior to submission.

Disclaimer The views and opinions expressed therein are those of the authors and do not necessarily reflect those of the Health Technology Assessment Programme, NIHR, NHS or the Department of Health.

Open Access This is an Open Access article distributed in accordance with the terms of the Creative Commons Attribution (CC BY 4.0) license, which permits others to distribute, remix, adapt and build upon this work, for commercial use, provided the original work is properly cited. See: http:// creativecommons.org/licenses/by/4.0/

\section{REFERENCES}

1. O'Brien PE, McPhail T, Chaston TB, et al. Systematic review of medium-term weight loss after bariatric operations. Obes Surg 2006; $16: 1032-40$.

2. Picot J, Jones J, Colquitt JL, et al. The clinical effectiveness and cost-effectiveness of bariatric (weight loss) surgery for obesity: a systematic review and economic evaluation. Health Technol Assess 2009;13:1-357, iii.

3. Courcoulas AP, Yanovski SZ, Bonds D, et al. Long-term outcomes of bariatric surgery: a National Institutes of Health Symposium. JAMA Surg 2014;149:1323-9.

4. Welbourn R. National bariatric surgery registry: UK internet-based surgery outcomes database. Obes Surg 2009;19:993.

5. Belle SH, Berk PD, Courcoulas AP, et al. Safety and efficacy of bariatric surgery: Longitudinal Assessment of Bariatric Surgery. Surg Obes Relat Dis 2007;3:116-26.

6. Information Services Division National Services Scotland. Assessment of SMR01 Data 2010-2011. 2012. http://www. isdscotland.org/Health-Topics/Hospital-Care/Publications/2012-05 08/Assessment-of-SMR01Data-2010-2011-ScotlandReport.pdf

7. Shikora SA, Kim JJ, Tarnoff ME, et al. Laparoscopic Roux-en-Y gastric bypass: results and learning curve of a high-volume academic program. Arch Surg 2005;140:362-7.
8. Mercer SW, Watt GCM. The inverse care law: clinical primary care encounters in deprived and affluent areas of Scotland. Ann Fam Med 2007;5:503-10.

9. Wadden TA, Foster GD. Weight and Lifestyle Inventory (WALI). Surg Obes Relat Dis 2006:2:180-99.

10. Ware J, Kosinski M, Keller SD. A 12-Item Short-Form Health Survey construction of scales and preliminary tests of reliability and validity. Med Care 1996;34:220-33.

11. Williams A. EuroQol-a new facility for the measurement of health-related quality of life. Health Policy (New York) 1990;16:199-208.

12. Spitzer RL, Kroenke K, Williams JB. Validation and utility of a self-report version of PRIME-MD: the PHQ primary care study. Primary Care Evaluation of Mental Disorders. Patient Health Questionnaire. JAMA 1999;282:1737-44.

13. Spitzer RL, Kroenke K, Williams JBW, et al. A brief measure for assessing generalized anxiety disorder: the GAD-7. Arch Intern Med 2006;166:1092-7.

14. Kolotkin RL, Crosby RD. Psychometric evaluation of the impact of weight on quality of life-lite questionnaire (IWQOL-Lite) in a community sample. Qual Life Res 2002;11:157-71.

15. Derby CA, Araujo AB, Johannes CB, et al. Measurement of erectile dysfunction in population-based studies: the use of a single question self-assessment in the Massachusetts Male Aging Study. Int J Impo Res 2000;12:197-204.

16. Barry MJ, Fowler FJ, O'Leary MP, et al. The American Urological Association symptom index for benign prostatic hyperplasia. The Measurement Committee of the American Urological Association. $J$ Urol 1992;148:1549-57; discussion 1564.

17. Abrams P, Avery K, Gardener N, et al. The international consultation on incontinence modular questionnaire: http://www.iciq.net. J Urol 2006;175:1063-6.

18. Macran S, Wileman S, Barton G, et al. The development of a new measure of quality of life in the management of gastro-oesophageal reflux disease: the Reflux questionnaire. Qual Life Res 2007; 16:331-43.

19. Babor TF, Higgins-Biddle JC, Saunders JB, et al. The alcohol use disorders identification test guideline for use in primary care. World Health Organization, Department of Mental Health and Substance Dependence, 2001. http://whqlibdoc.who.int/hq/2001/WHO_MSD_ MSB_01.6a.pdf?ua=1

20. Craig CL, Marshall AL, Sjöström M, et al. International physical activity questionnaire: 12 -country reliability and validity. Med Sci Sports Exerc 2003;35:1381-95.

21. Roe L, Strong C, Whiteside C, et al. Dietary intervention in primary care: validity of the DINE method for diet assessment. Fam Pract 1994;11:375-81.

22. Scheier MF, Carver CS, Bridges MW. Distinguishing optimism from neuroticism (and trait anxiety, self-mastery, and self-esteem): a reevaluation of the Life Orientation Test. J Pers Soc Psycho 1994;67:1063-78.

23. Foster GD, Wadden TA, Vogt RA, et al. What is a reasonable weight loss? Patients' expectations and evaluations of obesity treatment outcomes. J Consult Clin Psychol 1997;65:79-85.

24. Health Protection Scotland. The epidemiology of orthopaedic surgical site infection occurring up to one year after surgery: a feasibility study of telephone screening and direct observation by trained healthcare workers. Health Protection Scotland, 2010. http:// www.documents.hps.scot.nhs.uk/hai/sshaip/publications/ssi/ ortho-ssi-report-2010.pdf

25. Ertlet TW, Marino JM, Mitchell JE. Psychosocial aspects of body contouring surgery after bariatric surgery. 2010:633-9.

26. Keating CL, Moodie ML, Bulfone L, et al. Healthcare utilization and costs in severely obese subjects before bariatric surgery. Obesity (Silver Spring) 2012;20:2412-19.

27. Bruce J, Thornton AJ, Scott NW, et al. Chronic preoperative pain and psychological robustness predict acute postoperative pain outcomes after surgery for breast cancer. $\mathrm{Br} J$ Cancer 2012;107:937-46. 